\title{
Civilisations
}

Revue internationale d'anthropologie et de sciences

humaines

$57 \mid 2008$

Tourisme, mobilités et altérités contemporaines

\section{Hilma Granqvist, Louise Baldensperger et la " tradition de rencontre » au village palestinien d'Artas}

\section{Falestin Naïli}

\section{(2) OpenEdition}

Journals

\section{Édition électronique}

URL : http://journals.openedition.org/civilisations/1201

DOI : 10.4000/civilisations. 1201

ISSN : 2032-0442

\section{Éditeur}

Institut de sociologie de l'Université Libre de Bruxelles

\section{Édition imprimée}

Date de publication : 29 décembre 2008

Pagination : 127-138

ISBN : 2-87263-022-8

ISSN : 0009-8140

\section{Référence électronique}




\title{
Hilma Granqvist, Louise Baldensperger et la «tradition de rencontre» au village palestinien d'Artas
}

\author{
Falestin NAÏLI
}

Résumé : Cet article analyse la manière dont l'œuvre et la mémoire de l'anthropologue finlandaise Hilma Granqvist et de son hôtesse française Louise Baldensperger ont été intégrées, en Palestine, dans un projet de patrimonialisation du passé paysan du village d'Artas, la localité où le travail ethnographique de Granqvist prit place pendant les années du Mandat britannique sur la Palestine. Ce projet de patrimonialisation a inscrit la mémoire des deux Européennes dans une " tradition de rencontre " locale, dans un souci d'attirer des touristes et des agences de coopération internationales.

Mots-clés : mémoire, patrimoine, Palestine, Hilma Granqvist, Louise Baldensperger.

Abstract: This article presents an analysis of the way in which the work and the memory of Finnish anthropologist Hilma Granqvist and of her French hostess Louise Baldensperger have been incorporated in Palestine in a cultural heritage project on the peasant past of the village of Artas, where Granqvist's ethnographic fieldwork took place at the time of the British Mandate. This cultural heritage project inscribed the memory of these two European women in a local "tradition of encounter", in order to try to attract tourists and international cooperation agencies.

Key words: memory, cultural heritage, Palestine, Hilma Granqvist, Louise Baldensperger. 


\section{A}

début du $20^{\mathrm{e}}$ siècle, l'anthropologue finlandaise Hilma Granqvist choisit le village d'Artas près de Bethléem pour une étude approfondie de la société villageoise palestinienne. Accueillie au village et aidée par Louise Baldensperger, la fille d'un missionnaire alsacien, Granqvist y entreprend une étude ethnologique qui sera publiée sous la forme de cinq monographies consacrées aux « trois grands événements de la biographie humaine » (Granqvist 1965 : 9) : naissance, mariage et mort. Avec Louise Baldensperger, Granqvist occupe jusqu'à aujourd'hui une place importante dans la mémoire collective des villageois et joue un rôle prépondérant dans un projet local de patrimonialisation du passé paysan. En effet, en 1993, Mūsā Sanad, un habitant du village, a fait de l'œuvre de Granqvist le point de départ de l'établissement d'un Centre du Patrimoine Populaire Palestinien à Artas. Pourquoi Mūsā Sanad s'est-il attaché à l'œuvre et à la mémoire de Granqvist, ainsi qu'à celle de Louise Baldensperger ? Comment les a-t-il utilisées pour attirer l'intérêt des touristes et des agences de coopération? Ce seront les principales questions auxquelles cet article tentera de répondre, en proposant une double analyse des activités de ce Centre et des écrits de Mūsā Sanad. L'article aborde donc la question de l'impact de la recherche anthropologique sur le terrain et celle de son usage par ceux qui en constituaient l'objet, à travers l'utilisation de l'héritage de Hilma Granqvist et Louise Baldensperger dans le double contexte du tourisme et du développement. Derrière ces deux domaines se profile la question de la représentation de l'identité palestinienne, représentation contestée et politisée, ainsi que celle de la signification du passé palestinien pour l'avenir de cet état en suspend.

\section{Artas : histoire(s) et mémoire(s)}

Le village d'Artas se trouve dans une vallée fertile à seulement trois kilomètres au sud de Bethléem. Très majoritairement musulmane, sa population est aujourd'hui d'environ 3000 habitants. Le nom d'Artas est un dérivé du latin Hortus conclusus, nom donné à la vallée par les Croisés en raison de son histoire biblique supposée ${ }^{1}$. D'après certaines recherches bibliques, la vallée d'Artas pourrait être l'emplacement du jardin clos du Roi Salomon, connu sous le nom d'Etham. Cette hypothèse a en tout cas favorisé l'installation de colons millénaristes et de missionnaires européens et américains dans la vallée au milieu du $19^{\mathrm{e}}$ siècle. Le premier colon européen à s'installer à Artas était John Meshullam, un Anglais d'origine juive converti au christianisme. Sa colonie agricole avait le soutien du Consul britannique de l'époque, James Finn, ainsi que celui des milieux millénaristes européens et américains. John Meshullam et ses associés concevaient cette colonie comme une préparation du terrain pour le « retour» du Christ, attendu pour le millénaire, par le biais de l'enseignement aux juifs appauvris de Jérusalem de l'agriculture et du christianisme. Pendant plus de deux décennies, la colonie a suscité des réactions complexes de la part de la population locale (villageois et bédouins des alentours), allant de l'arrangement au refus violent.

Dans la mémoire collective des villageois, elle n'a pas laissé de traces, bien qu'il reste un indice toponymique dans la vallée d'Artas. Le nom d'Henri Baldensperger (appelé Anari par les villageois), un missionnaire alsacien qui fut l'associé de John Meshullam

1. En face du village, il y a un couvent catholique qui a adopté le nom latin. 
pendant moins de deux ans, avant de travailler à l'école protestante de l'évêque Gobat à Jérusalem, est en effet toujours connu à quelques villageois âgés. Toutefois, on ne se rappelle de lui qu'en lien avec sa fille Louise Baldensperger, cette dernière occupant une place importante dans la mémoire collective des villageois, avec Hilma Granqvist.

\section{Louise Baldensperger : l'hôtesse dans les jardins de Salomon}

Louise Baldensperger (1862-1938) se considérait comme une scientifique amateur (Fitch 1930 : 11). Dans sa maison d'Artas, elle accueillait des chercheurs en études bibliques, en archéologie, en botanique et en anthropologie (Barghouthi 1987 : 25). Elle n'a écrit qu'un seul livre en collaboration avec la folkloriste britannique Grace M. Crowfoot, mais elle a contribué à plusieurs études folkloriques et anthropologiques en tant que conseillère. Hilma Granqvist a particulièrement apprécié l'aide et la contribution de Louise Baldensperger à son travail (Granqvist 1931 : 19).

Partageant son temps entre Jérusalem et Artas, Louise Baldensperger s'est toujours considérée comme une locale, sans toutefois se fondre dans la population du village. Dans l'introduction du livre From Cedar to Hyssop ${ }^{2}$ qu'elle écrit avec Grace Crowfoot, elle signe : «Louise Baldensperger, une fille de Sion habitant les jardins de Salomon ». Elle note la date du 18 mai 1931, mais aussi : " Muharram, nouvelle année musulmane ${ }^{3}$ ». Sa manière de s'identifier était donc clairement biblique : elle se voyait comme une descendante des Hébreux (probablement en termes spirituels) qui habitait les jardins de Salomon. L'espace était biblique, mais le temps ne l'était pas puisqu'elle précise la date du calendrier musulman. Même si la Palestine était pour elle définie par son lien avec la Bible, elle avait parfaitement conscience du fait qu'elle y vivait à l'époque islamique.

À Artas le souvenir de Louise Baldensperger s'est transmis de la génération de ses voisins villageois aux générations suivantes, l'inscrivant dans la mémoire collective avec son appellation locale de Sitt Luīz. Son souvenir est lié à celui de Hilma Granqvist, qui est connue sous l'appellation locale Sitt Halìma, ce qui en dit long sur le respect et l'appréciation que lui témoignaient les villageois ${ }^{4}$. Le fait que ces derniers leur aient attribué à toutes les deux un nom arabe, précédé par le terme respectueux de Sitt (semblable à Madame), démontre leur volonté de les intégrer dans la communauté villageoise, et ce, même rétrospectivement. À Artas, ces deux étrangères ne sont donc pas connues sous leur nom européen mais surtout et, presque exclusivement, sous leur nom d'adoption. Les activités du Centre du Patrimoine Populaire Palestinien ont renforcé la mémoire de ces deux Européennes, et, comme nous allons le montrer, l'ont investie d'une signification précise.

\section{Hilma Granqvist: anthropologue pionnière et "gardienne de la mémoire »}

Hilma Granqvist (1890-1972) a écrit cinq monographies basées sur ses enquêtes ethnographiques à Artas, dont la plus grande partie se déroula pendant les années du

2. From Cedar to Hyssop est un livre sur le folklore des plantes en Palestine.

3. Barghouthi, 1987, p. 27, ma traduction.

4. Le surnom Sitt Halìma rappelle Halìma, la nourrice du prophète Muhammad. 
mandat britannique 5 . La particularité de ses livres est sans doute le fait qu'on y entend parler ses interlocuteurs villageois (en majorité des femmes), dans des longues citations, et qu'on y trouve leurs explications de leurs pratiques sociales, et non seulement celles de l'anthropologue. L'œuvre de Granqvist était reconnue par d'éminents anthropologues de son époque, comme Margaret Mead et Evans-Pritchard, mais, pour des motifs divers, le milieu académique finlandais n'était pas prêt à l'accueillir et elle n'y a jamais fait carrière. L'une des raisons de ce rejet était que Granqvist avait rompu avec le modèle orientaliste classique comme cadre sine qua non des études sur la Palestine, et qu'elle avait fait certains choix méthodologiques sortant du cadre du comparatisme britannique, tendance majoritaire de l'école d'anthropologie de l'époque (Isotalo 1995) ${ }^{6}$. Or, c'est précisément cette rupture qui rend son travail moderne et qui a, depuis les années 1990, justifié l'engouement renouvelé pour son œuvre surtout dans les pays scandinaves et anglophones.

Hilma Granqvist critiquait la littérature existante sur la Palestine pour ses lacunes sur le plan de l'ethnographie et pour son manque d'exactitude dus en partie, selon elle, à l'association trop étroite entre les coutumes contemporaines palestiniennes et celles de la Bible, plus précisément celles de l'Ancien Testament. Si elle décelait des parallèles entre les coutumes des Palestiniens contemporains et les coutumes bibliques, elle portait un regard critique sur la création d'un lien trop étroit entre les deux. Elle estimait que beaucoup de chercheurs de son époque avaient succombé à ce qu'elle appelle le « danger biblique » (Granqvist 1931 : 9). Granqvist n'a pas pour autant complètement rompu avec l'orientalisme classique et son esprit exégétique. Elle a ainsi notamment souligné les parallèles entre les coutumes qu'elle observait et les coutumes des peuples de l'Ancien Testament, notamment dans l'index du deuxième tome de son livre, Marriage Conditions in a Palestinian Village (Moors 1997 : index non paginé). En somme, l'œuvre de Hilma Granqvist représente, selon l'expression d'Annelies Moors, une « combinaison de critique et d'accommodation » (Moors 1997 : index non paginé).

Pour les folkloristes palestiniens, cette œuvre est d'une grande importance, car elle représente un inventaire très détaillé des pratiques sociales avant l'année 1948 et donc un lien avec la société palestinienne telle qu'elle était avant son éclatement. L'anthropologue Sharif Kanaana ${ }^{7}$, professeur à l'Université de Birzeit, est un des fondateurs du mouvement folklorique palestinien, qui s'est constitué, dans sa forme moderne, dans les années 1970. Il a également été membre du premier conseil d'administration du Centre du Patrimoine Populaire Palestinien à Artas. Dans une présentation qu'il a faite à l'occasion d'une

5. Granqvist choisit le village d'Artas pour son travail de terrain, d'une part, à cause de la proximité de Jérusalem où elle prenait des cours d'arabe et, d'autre part, parce qu'elle savait que Louise Baldensperger y habitait et y accueillait des chercheurs occidentaux.

6. Voir au sujet de l'œuvre de Granqvist : Naïli, F. «L'œuvre de Hilma Granqvist : L'Orient imaginaire confronté à la réalité d'un village palestinien », Revue d'études palestiniennes, automne 2007, 105, pp. 74-84.

7. Sharif Kanaana est l'auteur de nombreux livres et articles en anthropologie, folklore et histoire orale, dont notamment Speak Bird, Speak Again : Palestinian Arab Folktales (avec Ibrahim Muhawi), Berkeley, University of California Press, 1989. Je le remercie de m'avoir envoyé le texte de cette présentation. 
conférence consacrée au travail de Hilma Granqvist en $1997^{8}$, il explique l'importance de son œuvre du point de vue palestinien :

The work of Hilma Granqvist among Palestinians [...] was, in a sense, prophetic. Her work was detailed, comprehensive, accurate and honest enough to give one the feeling she knew that Palestinians were going one day to need to fall back on their folklore and folk heritage. That day has now come. Today Palestinians, possibly more than any other people in the world, are anxious about their future, because they face conditions which threaten their identity and their very existence. [...] There is a need to transmit to Palestinian children, wherever they may be, a unifying core of Palestinian culture, in order to rehabilitate them and help them maintain their Palestinian identity. [...] if and when such a program is launched, in order to acquaint Palestinian children all over the world, with what the life of their fellahin ancestors in the villages of the pre-1948 catastrophe was like, it will have to draw significantly on the work of Hilma Granqvist (Kanaana 1997).

Pour Sharif Kanaana, l'œuvre de Granqvist requiert une immense valeur identitaire, car elle permet la compréhension de la vie des fallāhīn (agriculteurs) avant 1948. En effet, le mouvement folklorique palestinien place l'image du fallāh (agriculteur) au centre de l'identité palestinienne. Cette tendance est devenue majoritaire après 1948 car le paysan palestinien symbolise le « lien 'naturel' à la terre perdue » (Aubin 2006 : 116). C'est dans le cadre de cette approche que la réception et l'utilisation de l'œuvre de Granqvist à Artas doivent être analysées.

\section{Le « technicien de la mémoire » et l'invention de la tradition de rencontre}

À travers le travail du Centre du Patrimoine Populaire Palestinien à Artas (Markaz Artas li'l-Turāth al-Sha 'bì al-Filaștīñ ), le savoir ethnographique produit sur le village par Hilma Granqvist (1890-1972) a été (ré)intégré au savoir local en tant que folklore. Le rôle du fondateur du Centre, Mūsā Sanad (1949-2005), véritable « technicien de la mémoire » (Aubin 2006), dans ce processus d'intégration a été central. Mūsā Sanad était un maître d'école et également le neveu d'une des principales interlocutrices de Granqvist. Il affirmait que la particularité d'Artas, au regard d'autres villages palestiniens, résidait dans les nombreuses études approfondies des aspects de la vie sociale dont il avait fait l'objet, ce qui lui conférait, à ses yeux, un statut de village type ${ }^{9}$. L'œuvre de Hilma Granqvist constituait, pour lui, la base principale de cette revendication de particularité et d'exemplarité car elle offre l'image la plus complète de la vie en Palestine à cette époque ${ }^{10}$. À l'instar des folkloristes palestiniens, Mūsā Sanad considérait l'œuvre de Granqvist comme une contribution importante à l'inventaire du folklore palestinien.

8. La conférence était intitulée In the Footsteps of Hilma Granqvist et a eu lieu à l'école Talita Kumi à Beit Jala en septembre 1997.

9. Signalons dans ce contexte que le village de Ban̄̄ Zayd a également fait l'objet de nombreux travaux sur le folklore pendant la période mandataire, mais que ces travaux ont été presque exclusivement l'œuvre de folkloristes palestiniens (Aubin $2004: 201-203$ ).

10. «Artas ka-namūdhaj li’l-qariya al-filastiniyya» (Artas comme modèle du village palestinien), une page, écrite par Mūsā Sanad, sans date. 
De plus, à travers son travail sur la généalogie des familles d'Artas, Granqvist avait également participé à la documentation sur l'enracinement des habitants d'Artas, élément central pour Mūsā Sanad.

L'inventaire du folklore et les arbres généalogiques s'inscrivent en effet dans le même registre mémoriel et identitaire. Le folklore permet la remémoration et la remise en scène perpétuelle d'une partie du mode de vie d'antan. La généalogie, quant à elle, fonde le lien des Palestiniens contemporains avec le monde de leurs ancêtres et donc avec leurs origines. Ce faisant, elle sert d'outil à la revendication d'antériorité des Palestiniens par rapport à la présence sioniste, ce qui fait d'elle un élément important du contre-discours palestinien face au discours sioniste. En conséquence, l'œuvre de Granqvist ne trouve toute sa signification pour les habitants contemporains d'Artas, et pour les Palestiniens en général, que lorsqu'elle est replacée dans le cadre de ce contre-discours palestinien dans lequel les activités de Mūsā Sanad s'inscrivent clairement. En effet, ce dernier pourrait entrer dans la catégorie de "l'intellectuel organique", au sens plus large que celui défini par Antonio Gramsci, car à travers son lien avec le mouvement folklorique, Mūsā Sanad était indirectement lié au mouvement nationaliste palestinien avec ses aspirations hégémoniques vis-à-vis de la sphère culturelle palestinienne et surtout vis-à-vis des représentations du passé palestinien ${ }^{11}$.

Mūsā Sanad disait de lui-même que sa vie avait pris un tournant décisif un jour de 1972 à l'occasion d'une rencontre avec une étrangère au village qui lui avait demandé son nom et montré ensuite son arbre généalogique, extrait du premier livre de Hilma Granqvist. La gêne qu'il ressentit face au savoir d'une étrangère plus étendu que le sien sur ses origines et sur l'histoire de son village natal à suscité son intérêt pour l'histoire d'Artas. Il a alors commencé à demander aux anciens du village de lui raconter leurs histoires et les a consignées par écrit. Il a également entrepris la mise à jour des arbres généalogiques créés par Granqvist, faisant appel à ses élèves pour les tenir à jour continuellement.

Jusqu'à sa mort en 2005, Mūsā Sanad a travaillé sur un manuscrit portant sur son village, en vue de publier un livre qui aurait abordé des sujets historiques autant qu'ethnographiques. Ce manuscrit constitue non seulement une source précieuse d'informations, mais il est surtout un miroir de l'image que Mūsā Sanad voulait projeter du passé et de l'avenir de son village. Il contient en effet des interprétations de certaines périodes de l'histoire d'Artas qui ne sont pas en accord avec ce que l'on peut constater dans les sources archivistiques. Ces différences sont particulièrement significatives au sujet de l'histoire de la présence précoce de colons européens et américains au village.

Mūsā Sanad écrit par exemple qu'au milieu du 19e siècle, «la part du lion de l'invasion (ghazu) des missions européennes en Palestine » est revenue à Artas. Un grand nombre d'Européens auraient acheté des terrains à Artas du fait " de leur amour intense pour la vallée fertile » et également pour les « anciennes ruines ». Ils auraient cultivé de nouvelles variétés de fruits et légumes. Il y a là un paradoxe entre la représentation de l'arrivée des Européens comme une invasion, référence possible au cadre plus large des aspirations colonialistes européennes, et celle de leurs motivations. L'amour du paysage, les perspectives d'engagement dans l'agriculture dans une région fertile et les anciennes

11. Ces aspirations hégémoniques, il est important de le rappeler, se placent dans un contexte où leur légitimité même est régulièrement remise en question car les Palestiniens vivent toujours sous domination israélienne. 
ruines - toutes ces motivations d'ordre général sont loin des objectifs spécifiques des millénaristes pour la « rédemption » de la Terre Sainte. L’identification des personnagesclés de cet épisode de l'histoire du village s'inscrit dans la même logique de généralisation mais elle semble même aller au-delà. Ainsi, Mūsā Sanad mentionne uniquement Peter Meshullam, le fils du fondateur de la colonie, et l'identifie comme un Allemand qui a acheté des terrains dans la vallée d'Artas. Par cette identification, Mūsā Sanad l'a, en fait, éloigné de la connexion anglo-sioniste qui a facilité la colonisation de la Palestine.

La subversion implicite dans cette présentation des faits devient tout à fait évidente lorsque Mūsā Sanad décrit l'installation des colons américains à Artas à partir de 1850 . Ce groupe était mené par une prophétesse millénariste auto-proclamée de Philadelphia, Clorinda Minor. Selon le manuscrit de Mūsā Sanad, les colons américains auraient fait construire une maison et y auraient établi un centre pour les activités agricoles et missionnaires ainsi que pour l'étude du folklore palestinien. En déclarant que la maison des colons servait également de centre d'étude du folklore palestinien, Mūsā Sanad a, de fait, situé le début des études folkloriques sur Artas avant la période de Louise Baldensperger et Hilma Granqvist. Il a également attribué une naissance plus précoce à la « tradition de rencontre » qu'il voit exemplifiée dans l'échange entre les deux Européennes et les villageois, ainsi que dans l'œuvre de Granqvist. Il a fait d'une période sombre de l'histoire d'Artas (sous la présence coloniale), un épisode renforçant la tradition de rencontre qu'il voulait promouvoir activement à travers le Centre du Patrimoine Populaire Palestinien à Artas. Il a ainsi préservé et mobilisé la mémoire de Hilma Granqvist et de Louise Baldensperger, dans un souci de recréer l'espace d'échanges et de rencontres que ces deux Européennes avaient, selon lui, initié avec leur voisins et interlocuteurs villageois. Le manuscrit de Mūsā Sanad fonctionne donc comme une "toile d'araignée », pour reprendre l'image utilisée par Liisa Malkki à propos de la formation et la transformation de ce qu'elle appelle la « conscience historique » (Malkki 1995 : 242). L'histoire présentée par Mūsā Sanad intègre certains éléments de l'histoire archivistique de son village, en omet d'autres et, enfin, transforme quelques éléments pour qu'ils prennent leur place dans ce récit historique.

Cette image de la toile d'araignée peut également s'appliquer au Centre du Patrimoine dont le programme est basé sur la notion de rencontre et de mise en pratique d'activités allant dans ce sens. Même après le décès de Mūsā Sanad, cette notion demeure centrale à la mission du Centre. Ainsi, pour la journée internationale de la femme le 8 mars 2007, le Centre a proposé une rencontre de femmes à Artas. Bien que l'annonce ait été rédigée par Leyla Zuaiter, consultante auprès du Centre du Patrimoine depuis le décès de Mūsā Sanad, elle s'inscrit dans la continuité des idées du fondateur :

If this village seems a bit out of the way for such an encounter, think again: since the 1840 's, no fewer than 10 foreign women of various nationalities have lived, worked, or studied in Artas, adding to the body of work about 'the most documented village in Palestine'. These women, some of whom have achieved legendary status, include a thwarted American millenarist, a British Consul's wife, a French missionary's daughter, a British botanist, Finnish and Canadian anthropologists, and a Swedish photographer ${ }^{12}$.

12. «The Artas Folklore Center Women's Group Celebrates a Century and a Half of Women's Encounters in Artas », This Week in Palestine (version internet), (107), mars 2007. 
Dans son manuscrit, Mūsā Sanad n'a fait aucune référence détaillée à la millénariste américaine Clorinda Minor ou à Elisabeth Finn, l'épouse du Consul britannique, car il ne voulait apparemment évoquer ni le nouvel ni l'ancien allié du mouvement sioniste en Palestine. Leyla Zuaiter, en évoquant Clorinda Minor et Elizabeth Finn, sans les nommer, dans l'annonce de la Rencontre des Femmes du 8 mars 2007, les intègre à la même lignée de femmes que Louise Baldensperger et Hilma Granqvist, qui étaient, elles, véritablement intéressées par la population villageoise. En effet, Leyla Zuaiter mobilise le souvenir de Clorinda Minor et d'Elizabeth Finn, qui étaient liées à la colonie agricole de John Meshullam, pour documenter, à l'instar de Mūsā Sanad, l'ancienneté de la tradition de rencontre à Artas. Nous ne savons pas si Leyla Zuaiter est pleinement consciente du cadre idéologique des activités de Clorinda Minor et d'Elizabeth Finn. Quoi qu'il en soit, dans le cadre de cette annonce, ces deux femmes servent d'exemple aux rencontres interculturelles et interreligieuses dans le but de renforcer la solidarité internationale avec les femmes palestiniennes. Elles sont donc intégrées à l'histoire d'Artas à travers le processus évoqué de la toile d'araignée. En revendiquant certains éléments de l'histoire du réseau millénariste organisé autour de la colonie de Meshullam, les nouveaux dirigeants du Centre se sont appropriés une autre partie de l'histoire d'Artas jusqu'alors marginalisée, et ils l'ont transformée pour mieux servir la cause du Centre.

\section{Le Centre du Patrimoine Populaire Palestinien : un lieu de mémoire et de rencontre}

Mūsā Sanad a fondé le Centre du Patrimoine Populaire Palestinien en 1993 en bénéficiant de l'aide et du soutien d'un conseil d'administration composé de personnalités très connues dans le domaine du folklore palestinien, dont Sharif Kanaana ${ }^{13}$. Ce Centre n'est pas consacré exclusivement au patrimoine ${ }^{14}$ tangible, c'est-à-dire aux objets et aux monuments; il est aussi engagé dans le domaine de ce qu'on appelle le patrimoine immatériel, comprenant l'histoire orale, les contes et les pratiques culturelles.

Le Centre porte deux noms différents en arabe et en anglais. Ces deux noms correspondent en fait à deux programmes et deux objectifs différents qui s'informent toutefois mutuellement. En arabe, le Centre s'appelle Markaz Artas li'l-Turāth al-Sha 'bì al-Filaștīñ ce qui veut littéralement dire Centre d'Artas pour le Patrimoine Populaire Palestinien. En anglais, il est nommé Artas Folklore Center ce qui peut être rendu par Centre Folklorique d'Artas.

13. Il s'agit notamment de Samīha Khalīl, fondatrice de l'association In 'āsh al-Usra, basée à Ramallah ; Abdullatif Al Barghouthi, professeur de langue arabe à l'Université de Birzeit; 'Abd al-'Azīz Abū Hadba, folkloriste palestinien, et Sharif Kanaana, anthropologue et professeur à l’Université de Birzeit.

14. J'utilise le terme patrimoine tout en soulignant que ce dont il est question à Artas n'est pas comparable au contexte historique français qui a donné naissance à une certaine conception du patrimoine, intimement lié à l'idée du bien public, ni au contexte plus généralement européen qui est depuis longtemps attaché à ce que Mariannick Jadé appelle « l'idéologie occidentale de la relique » (Jadé $2006: 20$ ). En effet, le terme arabe turāth dérive de waratha qui veut dire hériter, et se traduit par héritage, legs et patrimoine, selon les dictionnaires. La racine étymologique du terme turäth l'éloigne de la spécificité du patrimoine lié au concept du bien public. 
L'appellation arabe met le terme turāth au centre de la mission du centre. C'est un terme qui évoque, dans ce contexte, l'héritage culturel, généralement appelé patrimoine en français. Un objet (ou une pratique culturelle) défini par le mot patrimoine obtient une valeur symbolique dans le contexte de l'histoire d'une communauté ou d'une nation, étant appelé à témoigner de certains éléments de l'histoire considérés comme importants pour l'évolution et l'identité de cette communauté. Avec l'ajout de l'adjectif « populaire » au nom arabe du Centre d'Artas, ce patrimoine acquiert une caractéristique plus précise - il ne s'agit pas d'objets et de souvenirs d'une élite mais d'objets et de souvenirs de la masse populaire. Et enfin, avec l'ajout du mot «palestinien », ce patrimoine est attribué à une nation et sert alors la cause de l'affirmation de l'identité nationale. Le nom arabe du Centre l'inscrit donc pleinement à la fois dans le mouvement folklorique palestinien et dans le mouvement national palestinien, tous deux étroitement liés depuis 1948. Ce lien se retrouve par ailleurs dans une phrase de Mūsā Sanad, où il indique que l'un des objectifs d'un festival organisé annuellement par le Centre est d'encourager les paysans palestiniens à préserver « la terre et l'héritage de cette nation » (turāb waturāth hadhā al-watan). Dans la logique du mouvement folkloriste, que Mūsā Sanad rejoignait, le patrimoine ethnographique et le folklore se transmettent, tout comme la terre, de génération en génération, assurant la continuité du groupe en termes identitaires. Mūsā Sanad, en (re)intégrant le savoir ethnographique, constitué par Hilma Granqvist et ses interlocuteurs villageois, au folklore local s'est donc inscrit dans un mouvement qui dépasse largement les limites de son village.

Le lieu-phare du Centre est son musée ethnographique (Artas Ethnographic Museum), installé dans une maison historique au centre du village. En choisissant cette appellation pour le musée, Mūsā Sanad l'a intimement placé dans la lignée de l'œuvre ethnographique de Granqvist, affirmant ainsi la centralité du travail de l'anthropologue. Le patrimoine matériel exhibé au musée ethnographique - qui met en scène une maison paysanne d'il y a cent ans - ainsi que le patrimoine immatériel mis en scène pendant les soirées folkloriques font partie du patrimoine palestinien paysan. Le Centre reflète donc la communauté villageoise d'Artas telle qu'elle existait avant 1948, alors que les agriculteurs étaient largement majoritaires, mais il indique également un choix de la part de Mūsā Sanad. En effet, ce dernier privilégiait la culture paysanne dans sa représentation du patrimoine populaire palestinien, une démarche très répandue chez les folkloristes palestiniens.

Le nom anglais du Centre - Artas Folklore Center - met l'accent sur le terme «folklore » en omettant les mots palestinien et populaire. Ces deux adjectifs sont en retrait, semble-t-il, pour écarter une connotation politique qui pourrait décourager certains visiteurs étrangers. Aux yeux des touristes, le folklore rejoint le spectacle, la mise en scène et le divertissement. Dans ce contexte, une forme de spectacle folklorique est conçue pour le public n'appartenant pas à la communauté. Les programmes du Centre fabriqués pour les étrangers correspondent au moins en partie à cette acception du terme folklore. Ainsi, le Centre organise des visites guidées des lieux historiques dans les environs du village, suivies par des soirées avec un repas typique de la région, des chants et de la poésie traditionnelle. L'objectif de l'Artas Folklore Center réside donc dans sa participation au marché du tourisme alternatif en Palestine pour donner aux étrangers un « goût » (souvent littéralement) de la culture palestinienne et pour créer des opportunités économiques pour les villageois. Comme les activités du Markaz li'l-Turāth, celles de l'Artas Folklore Center produisent une représentation de l'identité palestinienne qui est 
fortement axée sur la culture paysanne. Celle-ci est donc toujours au centre, mais elle sert des objectifs différents.

\section{Les projets pour l'avenir et la mobilisation des ressources à travers la coopération}

Grâce au projet de Bethléem 2000 lancé par l'Autorité Palestinienne pour préparer Bethléem aux célébrations du millenium ${ }^{15}$ et à l'arrivée importante en Palestine de fonds de pays donateurs, Mūsā Sanad a vu la possibilité de réaliser à Artas de nombreux projets de rénovation, d'amélioration des infrastructures et de mise en valeur du paysage. Ainsi sont nés de nombreux accords de coopération avec des villes françaises et des organisations finlandaises mais aussi avec des organisations néerlandaises, belges et canadiennes. Les liens privilégiés que Mūsā Sanad a entretenus avec le Consulat Général de France à Jérusalem, avec le Bureau Représentatif de Finlande auprès de l'Autorité Palestinienne à Ramallah et avec d'autres organisations françaises et finlandaises nous intéressent ici particulièrement. Les relations étroites avec ces deux pays européens sont le résultat de la lecture sélective de l'histoire de la présence occidentale à Artas faite par Mūsā Sanad. Pour lui, les deux personnages clés de ce passé étaient Louise Baldensperger, une Française, et Hilma Granqvist, une Finlandaise. En établissant des liens solides avec les institutions représentatives de ces deux pays en Palestine, Mūsā Sanad a mobilisé la mémoire de ces deux femmes pour des projets visant à améliorer le présent et l'avenir de la communauté villageoise d'Artas.

Avec respect, le représentant de Cités Unies France (CUF) en Palestine de 1998 à 2002, a décrit Mūsā Sanad comme "Monsieur UNESCO d'Artas » en raison de la multitude de domaines dans lesquels Mūsā Sanad souhaitait intervenir, dont notamment l'éducation, la science (la recherche) et la culture. Je me limiterai ici à un seul exemple de projet franco-palestinien, à savoir le chantier de réhabilitation d'anciennes maisons d'Artas. En 1999, les villes de Grenoble, Vienne, Romans, Gières et Saint-Priest, en qualité de membres du Fonds de Coopération Décentralisée pour la Palestine (FCDP) de CUF, ont envoyé un groupe de jeunes à Artas pour un premier chantier « échanges patrimoines » dans le cadre duquel le groupe a œuvré à la rénovation d'une ancienne maison avec des bénévoles du village. L'attrait pour ces villes venait de l'idée qu'une Française, Louise Baldensperger, avait vécu dans ce village et qu'on pourrait, plus tard, réhabiliter sa maison, dans le cadre du chantier. Mūsā Sanad avait des projets ambitieux pour la réhabilitation des maisons : il voulait faire d'Artas un lieu phare du tourisme et souhaitait installer une salle d'exposition permanente ainsi qu'un restaurant dans deux des maisons choisies pour être réhabilitées. Dans la maison de Louise Baldensperger, il projetait d'établir un centre d'accueil pour les chercheurs étrangers, perpétuant ainsi la tradition d'hospitalité de Louise Baldensperger ${ }^{16}$.

15. Le projet de Bethléem 2000, avec un budget de deux cents millions de dollars a été soutenu et financé par la communauté internationale, et notamment par le Programme des Nations Unies pour le Développement, la Banque mondiale et la Communauté européenne (Andezian 2006 : 155).

16. En 2007, ces projets ambitieux n'ont pas encore vu le jour dû à la situation d'exception qui perdure dans les territoires occupés depuis le début de la deuxième Intifada en automne 2000. 
Un autre projet ambitieux implique l'Institut Finlandais au Moyen-Orient (FIME) et fait partie de l'une des activités de l'Institut dénommée « Integrated Development of Rural Communities in Palestine ». Ce projet a réuni le FIME, le Centre du Patrimoine d'Artas, le conseil du village et l'ONG palestinienne Applied Research Institute Jerusalem (ARIJ) autour de la création d'une base de données complète sur Artas, couvrant tous les aspects physiques, sociaux et économiques de la vie au village ${ }^{17}$. Dans le cadre de ce projet, la mémoire de Hilma Granqvist a été mobilisée par le FIME et par le Centre du Patrimoine. Le FIME, qui avait déjà essayé de monter un vaste projet d'étude d'Artas à partir de l'œuvre de Granqvist en 1999-2000 ${ }^{18}$, a justifié son engagement particulier dans ce village par la mémoire de l'anthropologue :

\begin{abstract}
Why Artas? Finland has a historic interest on Artas because of the famous anthropologist, Hilma Granqvist, who spent several years in the village with Sitt Louisa and published a set of important monographs on life there. Her work is well known among scholars as pioneering ethnography and represents a unique view on rural Palestinian society and especially on the life of Palestinian women in the village before the Second World War. Besides the ties between the village of Artas and Finland created by "Halime" the site is particularly suitable for this type of project because of its practical location near Bethlehem and Jerusalem, its relatively small size [...] and its well defined geographic location in a deep, wellwatered valley at the edge of the desert ${ }^{19}$.
\end{abstract}

Ces deux exemples montrent à quel point la mémoire de Hilma Granqvist et Louise Baldensperger a pu motiver les organisations impliquées dans ces projets à Artas. Cette mémoire n'était pas seulement un point de départ important dans le travail de Mūsā Sanad, mais constituait aussi le point d'ancrage de ses partenaires français et finlandais. «L'intérêt historique » auquel fait référence la dernière citation crée donc des nouveaux liens et des nouvelles rencontres, en s'inscrivant dans la lignée de Hilma Granqvist et Louise Baldensperger.

\title{
Conclusion
}

Dans le contexte palestinien, le tourisme a acquis une importante dimension politique, car il s'agit comme ailleurs de présenter le pays, son histoire et sa culture à « l'Autre », mais cet «Autre » a probablement été plus exposé au récit sioniste concernant la Palestine. Les acteurs palestiniens du tourisme ont donc pour tâche de défaire certains mythes créés par les idéologues sionistes et repris par les acteurs israéliens du tourisme. Ainsi, l'image du pionnier sioniste qui fait fleurir le désert est contrecarrée par celle du paysan palestinien qui travaille son champ. Cette image, qui est centrale au discours identitaire palestinien, trouve un appui important dans l'œuvre de Hilma Granqvist.

Hilma Granqvist, en donnant la parole à ses interlocuteurs villageois, a offert une documentation sans pareil d'une communauté villageoise palestinienne avant l'année 1948. Pourtant tel n'était pas son objectif : comme tous les anthropologues de son époque,

17. Finnish Institute in the Middle East, Artas Project 2001.

18. Finnish Institute in the Middle East, Project Description: Artas - Profile of a Palestinian Village II. Ce projet n'a jamais vu le jour car le financement n'a pas été trouvé.

19. Finnish Institute in the Middle East, Project Description: Artas - Profile of a Palestinian Village II. 
Hilma Granqvist n'écrivait pas pour ceux dont elle décrivait la vie dans ses monographies, à savoir les Palestiniens, mais pour des lecteurs européens intéressés par les sociétés arabes ou par le pays de la Bible. Bien qu'elle eût des contacts avec les folkloristes palestiniens de son époque, elle ne présageait probablement pas qu'un jour, ses livres occuperaient une place si importante à Artas et en Palestine en général. Ainsi, les folkloristes palestiniens modernes ont pu utiliser son œuvre dans le cadre de leur discours identitaire attribuant une place proéminente à la figure du fallāh palestinien. Mūsā Sanad, quant à lui, a su adapter l'œuvre de Granqvist pour qu'elle serve les besoins actuels de la communauté villageoise d'Artas : l'affirmation de son identité face à l'adversité, et la création d'opportunités économiques à travers le tourisme et la coopération internationale.

Pour Mūsā Sanad et pour le Centre du Patrimoine Populaire Palestinien à Artas, la mémoire de Hilma Granqvist et de Louise Baldensperger permet d'ancrer une tradition de rencontre dans le passé, même si cela implique une lecture sélective de l'histoire du village. Dans une réalité marquée par une occupation militaire de facto et une spoliation continuelle des terres du village au profit des colonies juives avoisinantes, l'invention de cette tradition constitue le signe d'un optimisme malgré tout.

\section{Références citées}

Andezian, Sossie, 2006. « Mémoires religieuses et construction nationale, Bethléem entre lieu de mémoire chrétien et ville autonome palestinienne », in Nadine Picaudou (dir.), Territoires palestiniens de mémoire, pp. 139-171. Paris : Karthala/ IFPO.

Aubin-Boltanski, Emma,

2004. Prophètes, héros et ancêtres : les pèlerinages musulmans de Nabî Mûsâ et de Nabî Sâlih dans la construction nationale palestinienne, thèse de doctorat, Paris, EHESS.

2006. " Le folkloriste comme technicien de la mémoire », in Nadine Picaudou (dir.), Territoires palestiniens de mémoire, pp. 115 -137. Paris : Karthala/ IFPO.

Barghouthi, Abdullatif, 1987. Arab Folk Stories from Artas. Bir Zeit: Birzeit University.

Fiтch, Florence Mary, 1930. “An A.A.U.W. Fellow in Palestine”, A.A.U.W. Journal, pp. 10-13.

GranQVist, Hilma,

1931. Marriage Conditions in a Palestinian Village, I. Helsinki : Societas scientiarum fennica, commentationes humanarum litterarum.

1965. Muslim Death and Burial: Arab Customs and Traditions Studied in a Village in Jordan. Helsinki: Societas Scientiarum Fennica, Commentationes Humanarum Litterarum.

Isotalo, Riina, 1995. "Edward Westermarck and Hilma Granqvist in the field of Orientalist discourse in Finland", Third Nordic Conference on Middle Eastern Studies, Joensuu, Finlande, juin 1995.

JADÉ, Mariannick, 2006. Le Patrimoine Immatériel, Perspectives d'interprétation du concept de patrimoine. Paris : L'Harmattan.

KanaAna, Sharif, 1997. "Granqvist, Folklore and the Palestinian National Identity", présentation donnée lors de la conférence intitulée, In the Footsteps of Hilma Granqvist, tenue à l'école Talita Kumi à Beit Jala, 3-7 septembre 1997.

Malkki, Liisa, 1995. Purity and Exile: Violence, Memory, and National Cosmology Among Hutu Refugees in Tanzania. Chicago: University of Chicago Press.

Moors, Annelies, 1997. "Visions of Palestine, Visualizing Women in Palestine: Hilma Granqvist's Published Photographs", présentation donnée lors de la conférence intitulée, In the Footsteps of Hilma Granqvist, tenue à l'école Talita Kumi à Beit Jala, 3-7 septembre 1997. 